\title{
Hemoglobin Genotype Polymorphism in Gravidas Women Attending Federal Medical Center Yola, Nigeria
}

\author{
Etim EA ${ }^{1 *}$, Adjekuko OC ${ }^{2}$ Ahmadu BU ${ }^{3}$ and Nweke $\mathrm{JN}^{4}$ \\ ${ }^{1}$ Hematology Department, Federal Medical Centre Yola, Nigeria \\ ${ }^{2}$ Department of Medical Laboratory Services, General Hospital Okwe, Nigeria \\ ${ }^{3}$ Emergency Pediatric Unit Federal Medical Center Yola, Nigeria \\ ${ }^{4}$ Hematology Department, Ambrose Alli University, Nigeria
}

*Corresponding author: EtimEA, Hematology Department, Federal Medical Centre Yola, Nigeria

\begin{abstract}
Background/Objective: Hemoglobin is the iron-containing oxygen-transport metalloprotein in our red blood cells, hemoglobin is made up of globin chains which are encoded by their respective genes located on chromosome 11 and16 with several alleles. Many of these alleles suffer point mutations in the DNA sequence that lead to single amino acid substitutions in the globin moiety, resulting in the production of abnormal hemoglobin polymorphism and which are associated with a wide range of moderate to severe hemolytic anemia in pregnant women. Therefore, this study aims to examine hemoglobin genotype polymorphism among gravidas women in Yola.

Materials/Methods: 904 pregnant (i.e. gravida) women with age range of 18 to 41 years in the antenatal ward of the hospital participated in this study and $2 \mathrm{mls}$ of venous blood were aseptically collected from each participant into EDTA vacutainer. The hemoglobin genotype was determined within 5hours of blood collection using Helena electrophoresis tank.

Results: 750.3(83\%) of women had hemoglobin genotype of AA while 144.6(16\%) women had hemoglobin genotype of AS. In addition, hemoglobin genotype AC was seen in $9(1 \%)$ of the gravida's women while hemoglobin genotype SS and SC was not seen in this group of women within the study period.

Conclusion: Gene frequencies with regard to the hemoglobin genotype polymorphism in gravidas women has shown a general formula of AA > AS > AC indicating high prevalence of AA over AS and AC in Federal Medical Center Yola Nigeria.
\end{abstract}

Keywords: Hemoglobin polymorphism; gravidas women

\section{Introduction}

Hemoglobin is the iron-containing oxygen-transport metalloprotein in red blood cells of humans and most vertebrates. Hemoglobin in our blood carries oxygen from respiratory organs to the rest of the body, where it releases the oxygen to metabolize nutrients to generate energy that powers our body's physiology and collects the resultant carbon dioxide back to respiratory organs to be expelled from the body. Hemoglobin is made up of heme, which is the iron-containing portion, and globin chains, which are proteins and the globin protein consists of chains of amino acids. There are several different types of globin chains, such as: alpha, beta, delta, and gamma. Hemoglobin Polymorphism in this study refers to the occurrence of variety of hemoglobin types in pregnant (i.e. gravidas) women. Hemoglobin types include: a) Hemoglobin A ( $\mathrm{Hb} \mathrm{A}$ ) which makes up about 95\%-98\% of hemoglobin found in adults; it contains two alpha $(\alpha)$ chains and two beta $(\beta)$ protein chains[1].

b) Hemoglobin $\mathrm{S}(\mathrm{HbS}$ ) which is an abnormal hemoglobin with a single nucleotide substitution (GTG for GAG) in the gene for beta globin on short arm of chromosome 11, resulting in the replacement of a glutamic acid residue with valine at the sixth position of both (i.e. homozygous state) or single (i.e. heterozygous state) globin chain[2].

Hemoglobin $\mathrm{C}(\mathrm{HbC})$ is also an abnormal hemoglobin similar to $\mathrm{HbS}$ but in $\mathrm{HbC}$, lysine replaces glutamic in the globin chain. Deoxygenation of either $\mathrm{HbS}$ or $\mathrm{HbC}$ exposes valine or lysine residue on the surface of the molecule, which forms hydrophobic 
interactions with adjacent chains, the resulting polymers align into bundles, causing distortion of the RBC into a crescent or sickle shape, consequently, reduces flexibility and increase deformability, which hinders passage of the cell through narrow blood vessels[3] resulting in sickle cell episodes. Sickle cell disorders include the homozygous state for Hemoglobin S, or sickle cell anemia (SS), the heterozygous state for Hemoglobin S or the sickle cell trait (AS), and the compound heterozygote state of Hemoglobin S together with other hemoglobin variants such as $\mathrm{C}$ or D can result in hemoglobin $\mathrm{AC}[3]$.The globin chains are encoded by their respective genes located on chromosome 11 and chromosome 16 and are both known to have several alleles[4].Many of these alleles suffer point mutations in the DNA sequence that lead to single amino acid substitutions in the globin moiety, resulting in the production of hemoglobin polymorphism. The abnormal hemoglobin genotype occurs when an affected individual inherits mutated globin gene(s) such as hemoglobin S, C, D, and E from both parents. Abnormal hemoglobin genotypes are inherited in an autosomal codominant fashion and occur by different combinations[5]. Several abnormal hemoglobin genotypes have been discovered but the most commonly encountered abnormal hemoglobin genotypes among Nigerians include AS, AC, SC, and SS[5].It has been reported that abnormal hemoglobin genotypes have been associated with a wide range of moderate to severe hemolytic anemia, leading to a high degree of morbidity and mortality among affected individuals as well as susceptibility to renal medullary carcinoma[6]in addition, World Health Organization ranked Nigeria as first in terms of high prevalence of infants born with abnormal hemoglobin genotype[7]. Therefore, this study aims to examine hemoglobin genotype polymorphism among gravidas women in Yola in other to elucidate the risk of giving birth to children with abnormal hemoglobin as well as risk of developing hemolytic anemia during gestation period in this locality.

\section{Materials and Method}

This retrospective and descriptive study was carried out at the hematology department of Federal Medical Center Yola in Adamawa State, Northeastern Nigeria. 904 pregnant (i.e. gravida) women with age range of 18 to 41 years in the antenatal ward of the hospital participated in this study.

\section{Statistical analysis}

Statistical analysis was performed using SPSS computer software version 20.0 (IBM Chicago, IL, USA). Descriptive values were given as mean and standard error of mean. Categorical variables were expressed as the number of cases and the percentage value.

\section{Sample collection and analysis}

$2 \mathrm{mls}$ of venous blood were aseptically collected from each participant into a tripotassium Ethylenediaminetetraacetic acid (K3 EDTA) anticoagulant vacutainer. The hemoglobin genotype was determined within 5hours of blood collection as followsa portion of the blood was put in a clean khan tube and washed 3 times with normal saline $(0.85 \%$ sodium chloride). Distilled water was added to the washed red cell in ratio of 1:4 to lyse the blood sample. The lysed samples were applied on Helena cellulose acetate paper using the Helena plate and applicator, and the paper was placed in the Helena electrophoresis tank (Consort) containing a commercially prepared Tris-EDTA-Borate buffer, the $\mathrm{pH}$ of the buffer is 8.6. The electrophoretic separation was allowed at room temperature for 3minutes at 220V. A commercially prepared Helena known hemoglobin were run as controls along with the test, and the results were read immediately after the end of the test time.

\section{Results}

Hemoglobin genotype polymorphism in gravidas women have been analyzed and 750.3(83\%) of the women had hemoglobin genotype of AA while 144.6(16\%) women had hemoglobin genotype of AS. In addition, hemoglobin genotype AC was seen in $9(1 \%)$ of the gravidas women attending the antenatal clinic of federal medical center Yola as shown in Table 1.Age distribution shows that, $479(53 \%)$ and $54.2(6 \%)$ of hemoglobin genotype AA and AS respectively was seen in women within the age of 18 to 28years while $307.4(34 \%)$ of the hemoglobin genotype AA and $36.2(4 \%)$ of hemoglobin AS was observed in women within the age range of 29 to 39years. $1 \%$ of hemoglobin AC occurred in women within the age of 29 to 39years. In addition, among women at the age of 40 to 59years, 27(2.9\%) was observed at $\mathrm{P}<0.05$ as shown in Table 2. None of the gravidas women had hemoglobin SS or SC.

Table 1: Hemoglobin variants of gravidas women.

\begin{tabular}{|c|c|c|}
\hline Hemoglobin Variant & Number Observed & Percentage \\
\hline AA & 750.3 & 83 \\
\hline AS & 144.6 & 16 \\
\hline AC & 9 & 1 \\
\hline SS & 0 & 0 \\
\hline SC & 0 & 0 \\
\hline Total & 903.9 & 100 \\
\hline
\end{tabular}

Table 2: Hemoglobin variants per age group of gravidas women.

\begin{tabular}{|c|c|c|c|c|c|}
\hline \multirow{2}{*}{ Age Group } & \multicolumn{5}{|c|}{ Hemoglobin Variants } \\
\cline { 2 - 6 } & AA & AS & AC & SS & SC \\
& N (\%) & N (\%) & N (\%) & N (\%) & N (\%) \\
\hline $18-28$ & $479.1(53)$ & $54.2(6)$ & -- & -- & -- \\
\hline $29-39$ & $307.4(34)$ & $36.2(4)$ & 1 & -- & -- \\
\hline $40-59$ & $27(2.9)$ & -- & -- & -- & -- \\
\hline
\end{tabular}

\section{Discussion}

The analysis of hemoglobin polymorphism among pregnant women in Federal Center Yola revealed that gene frequencies with respect to the hemoglobin genotype polymorphism in gravidas women has shown a general formula of AA > AS > AC indicating high prevalence of AA over AS while AC genotype was the least of 
the hemoglobin genotype observed in this study. This result is in agreement with the earlier report by Medugu et al.[8] In addition, $83 \%$ of the women had hemoglobin genotype AA indicating that these women had normal allele of hemoglobin A in a homozygous state while $16 \%$ of women had hemoglobin genotype AS to reflect the presence of hemoglobin $\mathrm{A}(\mathrm{HbA})$ and abnormal hemoglobin $\mathrm{S}$ (HbS) in a heterozygous state and inherits one normal allele and one abnormal allele encoding hemoglobin S (hemoglobin genotype AS). Hemoglobin genotype AS is also called sickle cell trait which is generally regarded as benign condition but this condition have been reported to cause medical complications in exercise, muscle contraction or dehydrated state[9] and by consequence, $16 \%$ of pregnant women in this center may be at risk of anemia hence women with hemoglobin genotype of AS may require additional medical attention during vaginal child birth which usually involves levels of muscular contractions. Furthermore, 1\% of women had hemoglobin genotype AC and none of the women had genotype SS or $\mathrm{SC}$ and this low level of homozygous state of abnormal hemoglobin may be due to high level of medical education among couples or/ and that women with homozygous state of abnormal hemoglobin may be unable to keep pregnancy hence their absence in this study

\section{Conclusion}

Gene frequencies with respect to the hemoglobin genotype polymorphism in gravidas women has shown a general formula of AA > AS > AC indicating high prevalence of AA over AS and AC in Federal Medical Center Yola Nigeria.

\section{References}

1. Steinberg MH (2001) Modulation of fetal hemoglobin in sickle cell anemia. Hemoglobin 25:195-211.

2. Weatherall DJ, Clegg JB (2001) Inherited hemoglobin disorders: An increasing global health problem. Bull World Health Organ 79:704-712.

3. Adeyemi AA, Ladipo AO, Omolade OA, Ogbaro DD (2016) Frequency distribution of hemoglobin variants among teenagers. British journal of medicine \& research14(4):1-5.

4. Weatherall D (2011) The inherited disorders of hemoglobin: An increasing neglected global health burden. Indian J Med Res134(4):493497.

5. Fiekumo I Buseri, Charity N Okonkwo (2014) Abnormal hemoglobin genotypes and $\mathrm{ABO}$ and Rhesus blood groups associated with HIV infection among HIV-exposed infants in North Western Nigeria. Path Lab Med Intl(6):15-20.

6. Akigbe RE, Ige SF, Afolabi AO, Azeez OM, Adegunlola GJ (2009) Prevalence of haemoglobin variants, $\mathrm{ABO}$, and Rhesus blood groups in Ladoke Akintola University of Technology, Ogbomosho, Nigeria. Trends in Medical Research4(2):24-29.

7. (2006) World Health Organization. Sickle-Cell Anaemia. In: Fifty-Ninth World Health Assembly, Geneva, Switzerland.

8. Medugu JT, Abjah U, Nasir IA, Adegoke S, Asuquo EE (2016) Distribution of ABO, Rh D blood groups and hemoglobin phenotypes among pregnant women attending a Tertiary Hospital in Yola, Nigeria. J Med Trop 18:3842.

9. Tripette J, Loko G, Samb A, Gogh BD, Sewade E, et al. (2010) Effects of hydration and dehydration on blood rheology in sickle cell trait carriers during exercise. AJP. Heart and Circulatory Physiology299(3):908-914.
To Submit Your Article Click Here: Submit Article

DOI: $10.32474 /$ IPDOAJ.2020.04.000179

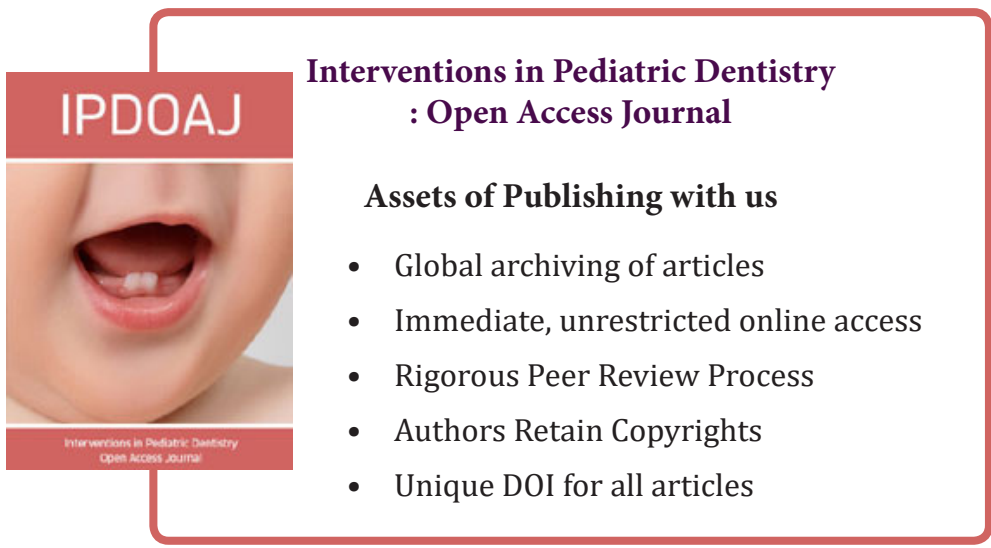

